\title{
2-酯基-1,5-苯并氧氮杂草的合成及反应副产物的研究
}

\author{
刘 倩 ${ }^{a}$ 赵利飞 ${ }^{a}$ 李文红 ${ }^{b}$ 李 媛 ${ }^{*}, a$ \\ ( ${ }^{a}$ 河北师范大学化学与材料科学学院 石家庄 050024) \\ $\left({ }^{b}\right.$ 河北工业职业技术学院环境与化学工程系 石家庄 050091)
}

\begin{abstract}
摘要 以取代苯、丁烯二酸䣶、邻氨基(对氯邻氨基)苯酚为原料, 合成了一系列 2-酯基-1,5-苯并氧氮杂草衍生物, 其结 构通过 IR, ${ }^{1} \mathrm{H}$ NMR, MS (HRMS)及元素分析进行表征. 同时, 确定了一个主要副产物 2-苯甲酰甲基-2H-1,4-苯并噁嗪3(4H)-䣳(6h')的结构, 提出了其可能的生成机理. 研究还表明, 中间体 4-芳基-4-氧代-2-丁烯酸酯(4)在对甲基苯磺酸为 催化剂、DMF 作溶剂、回流温度下反应时主要生成目标产物 2-酯基-1,5-苯并氧氮杂草 6, 而在冰醋酸为催化剂、甲醇 作溶剂、回流温度下反应时则主要生成副产物 $6 \mathbf{h}^{\prime}$.
\end{abstract}

关键词＼cjkstart迈克尔加成; 1,5-苯并氧氮杂草; 2-氨基苯酚; 4-氯-2-氨基苯酚; 反应机理

\section{Synthesis of 2-Ester-substituted-1,5-benzoxazepines and Studies on the By-product in the Reaction}

\author{
Liu, Qian ${ }^{a} \quad{\text { Zhao, } \text { Lifei }^{a} \quad \text { Li, Wenhong }}^{b} \quad \mathrm{Li}$, Yuan*,a \\ $\left({ }^{a}\right.$ College of Chemistry and Material Science, Hebei Normal University, Shijiazhuang 050024) \\ $\left({ }^{b}\right.$ Environment and Chemical Engineering, Hebei College of Industry and Technology, Shijiazhuang 050091)
}

\begin{abstract}
A series of new 1,5-benzoxazepines have been synthesized from substituted benzene, maleic anhydride and 2-aminophenol (4-chloro-2-aminophenol) and identified by IR, ${ }^{1} \mathrm{H}$ NMR, MS (HRMS) techniques and elemental analysis. Meanwhile, the structure of one main by-product 2-benzoylmethyl-2H-1,4-benzoxazin-3(4H)-one (6h') was identified, and the possible mechanism of producing the by-product was proposed. The optimization of reaction conditions showed that the reactions were performed in DMF solvent, at refluxed temperature and catalyzed by $p$-toluenesulfonic acid, and 2-ester-sub-stituted-1,5-benzoxazepines 6 were the main products. While, the reactions were performed in methyl alcohol, at refluxed temperature and catalyzed by acetic acid, and the by-product $\mathbf{6} \mathbf{h}$ ' was formed in good yield.
\end{abstract}

Keywords Michael addition; 1,5-benzoxazepine; 2-aminophenol; 4-chloro-2-aminophenol; reaction mechanism

氧氮杂草是新药创制中一类重要的活性物质, 具有 抗肿瘤活性 ${ }^{[1 \sim 4]}$ 、抗惊厥作用 ${ }^{[5]}$ 、杀菌 ${ }^{[6]}$ 等多种生物活性, 还能显著促进钾通道开放，促使血管及支气管扩张 ${ }^{[7]}$ 、 改善大脑缺血 ${ }^{[8]}$ 、抑制生物体内胆固醇合成, 从而起到 降血脂作用 ${ }^{[9 \sim 12]}$, 因此倍受药物化学和有机化学工作者 的关注. 本课题组在对 1,5 -苯并硫氮杂草生物活性的研 究中, 发现了一类对新生隐球菌等真菌的杀菌活性大大 超过抗真菌药物氟康唑的化合物. 该化合物活性高、对 细胞的毒性小, 且不同于氟康唑的作用靶点, 具有良好
的应用开发前景. 构效关系研究表明, 硫氮杂草的 $C(2)$ 上的乙酯基是此类硫氮杂草抗真菌药效团. 鉴于上述研 究结果以及氧氮杂草具有广谱生物活性这一特性, 我们 设计了一类 2-位酯基取代的 1,5-苯并氧氮杂草，以期发 现具有更好生物活性的氧氮杂草先导化合物. 本文合成 了 15 个 2 位酯基取代的 1,5 -苯并氧氮杂草衍生物，同时， 还研究了目标化合物的合成方法, 确定了一个主要副产 物的结构及其生成条件和机理. 目标化合物的合成路线 见 Scheme 1.

\footnotetext{
*E-mail: liyuanhbsd@163.com

Received May 29, 2012; revised July 11, 2012; published online July 17, 2012.

Project supported by the National Natural Science Foundation of China (No. 20972040).

国家自然科学基金(No. 20972040)资助项目.
} 
<smiles>[R]OC(=O)C=CC(=O)c1ccc([R])cc1</smiles><smiles>[X]c1ccc(O)c(N)c1</smiles><smiles>[R]C(=O)C(CC(=O)c1ccc([R1])cc1)Oc1ccc([X])cc1N</smiles><smiles>[R]C(=O)C1CC(c2ccc([R16])cc2)=Nc2cc([X])ccc2O1</smiles>

6a: $\mathrm{R}^{1}=\mathrm{OCH}_{3}, \mathrm{R}^{2}=\mathrm{CH}_{2} \mathrm{CH}_{3}, \mathrm{X}=\mathrm{H} ; \mathbf{6 b}: \mathrm{R}^{1}=\mathrm{H}, \mathrm{R}^{2}=\mathrm{CH}_{2} \mathrm{CH}_{3}, \mathrm{X}=\mathrm{H} ; \mathbf{6 c}: \mathrm{R}^{1}=\mathrm{CH}_{3}, \mathrm{R}^{2}=\mathrm{CH}_{2} \mathrm{CH}_{3}, \mathrm{X}=\mathrm{H}$; 6d: $\mathrm{R}^{1}=\mathrm{CH}_{2} \mathrm{CH}_{3}, \mathrm{R}^{2}=\mathrm{CH}_{2} \mathrm{CH}_{3}, \mathrm{X}=\mathrm{H}$; $\mathbf{6 e}: \mathrm{R}^{1}=\mathrm{F}, \mathrm{R}^{2}=\mathrm{CH}_{2} \mathrm{CH}_{3}, \mathrm{X}=\mathrm{H}$; $\mathbf{6 f}: \mathrm{R}^{1}=\mathrm{Cl}, \mathrm{R}^{2}=\mathrm{CH}_{2} \mathrm{CH}_{3}, \mathrm{X}=\mathrm{H}$; 6g: $\mathrm{R}^{1}=\mathrm{Br}, \mathrm{R}^{2}=\mathrm{CH}_{2} \mathrm{CH}_{3}, \mathrm{X}=\mathrm{H} ; \mathbf{6}$ : $\mathrm{R}^{1}=\mathrm{H}, \mathrm{R}^{2}=\mathrm{CH}_{3}, \mathrm{X}=\mathrm{H} ; \mathbf{6 i}: \mathrm{R}^{1}=\mathrm{H}, \mathrm{R}^{2}=\mathrm{CH}_{2} \mathrm{CH}_{2} \mathrm{CH}_{3}, \mathrm{X}=\mathrm{H}$; 6j: $\mathrm{R}^{1}=\mathrm{H}, \mathrm{R}^{2}=\mathrm{CH}\left(\mathrm{CH}_{3}\right)_{2}, \mathrm{X}=\mathrm{H}$; $\mathbf{6 k}: \mathrm{R}^{1}=\mathrm{H}, \mathrm{R}^{2}=\mathrm{C}\left(\mathrm{CH}_{3}\right)_{3}, \mathrm{X}=\mathrm{H} ; \mathbf{6 l}: \mathrm{R}^{1}=\mathrm{H}, \mathrm{R}^{2}=\mathrm{CH}_{3}, \mathrm{X}=\mathrm{Cl}$; 6m: $\mathrm{R}^{1}=\mathrm{H}, \mathrm{R}^{2}=\mathrm{CH}_{2} \mathrm{CH}_{3}, \mathrm{X}=\mathrm{Cl} ; \mathbf{6} \mathbf{n}: \mathrm{R}^{1}=\mathrm{H}, \mathrm{R}^{2}=\mathrm{CH}_{2} \mathrm{CH}_{2} \mathrm{CH}_{3}, \mathrm{X}=\mathrm{Cl} ; \mathbf{6}$ : $\mathrm{R}^{1}=\mathrm{H}, \mathrm{R}^{2}=\mathrm{CH}\left(\mathrm{CH}_{3}\right)_{2}, \mathrm{X}=\mathrm{Cl}$

\section{Scheme 1}

\section{1 结果与讨论}

\section{1 目标化合物的合成及波谱特征}

\subsection{1 合成}

由中间体 4 到目标化合物 6 经过两步反应, 首先邻 氨基苯酚与 $\alpha, \beta$-不饱和化合物 4 发生迈克尔加成, 之后 发生分子内缩合反应, 生成 2-酯基-1,5-苯并氧氮杂草衍 生物. 研究表明, 第二步关环反应比较容易进行, 第一 步反应需要酸催化. 本文以目标化合物 $\mathbf{6 1}$ 的合成为模型 反应，从催化剂、溶剂两方面对反应条件进行了系统优 化, 结果见表 1 .

表 1 催化剂、溶剂对化合物 $\mathbf{6 1}$ 产率的影响 ${ }^{a}$

Table 1 Effect of catalyst and solvent on the yield of the product $6 \mathbf{1}$

\begin{tabular}{|c|c|c|c|}
\hline Entry & Solvent & Catalyst/mol\% & Yield/\% \\
\hline 1 & $\mathrm{CH}_{3} \mathrm{OH}$ & 乙酸 $/ 40 \%$ & $15.7^{c}$ \\
\hline 2 & $\mathrm{C}_{6} \mathrm{H}_{6}$ & 乙酸 $/ 40 \%$ & $0^{d}$ \\
\hline 3 & $\mathrm{CH}_{3} \mathrm{CN}$ & 乙酸 $/ 40 \%$ & $0^{d}$ \\
\hline 4 & $\mathrm{HOCH}_{2} \mathrm{CH}_{2} \mathrm{OH}^{b}$ & 乙酸 $/ 40 \%$ & $0^{d}$ \\
\hline 5 & DMSO & 乙酸 $/ 40 \%$ & $0^{d}$ \\
\hline 6 & DMF & 乙酸 $/ 40 \%$ & 微量 ${ }^{d}$ \\
\hline 7 & $\mathrm{CH}_{3} \mathrm{OH}$ & 硅钨酸/1\% & 微量 ${ }^{d}$ \\
\hline 8 & $\mathrm{C}_{6} \mathrm{H}_{6}$ & 硅钨酸/1\% & $0^{d}$ \\
\hline 9 & $\mathrm{HOCH}_{2} \mathrm{CH}_{2} \mathrm{OH}^{b}$ & 硅铇酸/1\% & $0^{d}$ \\
\hline 10 & DMSO & 硅铇酸/1\% & 微量 ${ }^{d}$ \\
\hline 11 & DMF & 硅钨酸/1\% & $30.1^{c}$ \\
\hline 12 & DMF & 对甲苯磺酸 $/ 50 \%$ & $36.7^{c}$ \\
\hline 13 & $\mathrm{CH}_{3} \mathrm{OH}$ & 对甲苯磺酸/50\% & $0^{d}$ \\
\hline 14 & $\mathrm{C}_{6} \mathrm{H}_{6}$ & 对甲苯磺酸/50\% & $0^{d}$ \\
\hline 15 & $\mathrm{CH}_{3} \mathrm{OH}$ & 鏻钼酸/1\% & $0^{d}$ \\
\hline 16 & $\mathrm{C}_{6} \mathrm{H}_{6}$ & 鏻钼酸/1\% & $0^{d}$ \\
\hline 17 & DMF & 鏻钿酸/1\% & 微量 ${ }^{d}$ \\
\hline
\end{tabular}

${ }^{a}$ Reaction time: $8 \mathrm{~h}$, reaction temperature: reflux temperature. ${ }^{b}$ Reaction temperature: $130{ }^{\circ} \mathrm{C} .{ }^{c}$ Isolated yield. ${ }^{d}$ Monitoried by TLC.
从表 1 可以看出, 以冰乙酸为催化剂, 在甲醇溶液 中反应时, 杂草 $\mathbf{6 1}$ 的产率为 $15.7 \%$, 但在其他溶剂中几 乎得不到目标化合物. 用对甲基苯磺酸作催化剂, 在 DMF 中反应时产率最高(36.7\%). 杂多酸是一种固体强 酸, 催化迈克尔加成等其它缩合反应, 经常会得到很好 的效果. 于是, 本文还探索了硅铇酸或鏻钿酸催化 $\alpha, \beta$ 不饱和酮 4 与氨基苯酚的反应. 研究表明, 硅铇酸作催 化剂时, 在 DMF 中反应产率最高(30.1\%), 且硅铇酸的 用量仅为反应物 4 的 $1 \mathrm{~mol} \%$, 催化剂的用量再增加, 61 的产率没有大的变化. 在所考察的催化剂中, 鏻钿酸的 催化作用最差. 经分析对比, 最后选择了对甲基苯磺酸 作催化剂, DMF 作溶剂成功合成了其它目标化合物 $\mathbf{6}$.

表 2 化合物 $6 \mathbf{a} \sim 60$ 的产率 ${ }^{a}$

Table 2 The yields of compounds $\mathbf{6 a} \sim \mathbf{6 0}$

\begin{tabular}{cc||cc}
\hline Compd. & Yield $^{b} \%$ & Compd. & Yield $^{b} \%$ \\
\hline $\mathbf{6 a}$ & 21.3 & $\mathbf{6 i}$ & 30.1 \\
$\mathbf{6 b}$ & 32.6 & $\mathbf{6 j}$ & 24.6 \\
$\mathbf{6 c}$ & 31.8 & $\mathbf{6 k}$ & 4.5 \\
$\mathbf{6 d}$ & 30.8 & $\mathbf{6 1}$ & 36.7 \\
$\mathbf{6}$ & 32.3 & $\mathbf{6 m}$ & 34.5 \\
$\mathbf{6}$ & 32.8 & $\mathbf{6 n}$ & 30.5 \\
$\mathbf{6 g}$ & 34.1 & $\mathbf{6 o}$ & 26.3 \\
$\mathbf{6 h}$ & 32.6 & & \\
\hline
\end{tabular}

${ }^{a}$ Reaction conditions: $8 \mathrm{~h}$, reflux temperature, molar ratios of 4-aryl-4-oxo2-crotonates (4) to catalyst $\left(p-\mathrm{CH}_{3} \mathrm{C}_{6} \mathrm{H}_{4} \mathrm{SO}_{3} \mathrm{H}\right): 2: 1$ or $5: 1 ;{ }^{b}$ Isolated yield.

\subsection{2 波谱特征分析}

在 ${ }^{1} \mathrm{H}$ NMR 谱中, 目标化合物 $\mathbf{6 a} \sim \mathbf{6 0}$ 在化学位移 值 $(\delta) 8.04 \sim 6.85$ 处出现芳环氢的吸收峰, 分别代表 9 或 8 个氢, 在 $\delta 5.98 \sim 5.90\left(\mathrm{H}_{\mathrm{X}}\right), 2.87\left(\mathrm{H}_{\mathrm{A}}\right) \sim 2.40\left(\mathrm{H}_{\mathrm{B}}\right)$ 处出 现 12 重峰, 是典型的 $A B X$ 系统氢谱的特征, 为七元杂 环上的三个氢的化学位移值, 其偶合常数分别为 3.0 , 
$10.0,10.0,3.0 \mathrm{~Hz}$. 此外, $\mathrm{C}(2)$ 乙酯基取代的杂草 $\mathbf{6} \mathbf{a} \sim \mathbf{6 g}$ 在 $\delta \quad 1.25 \sim 1.26$ 处出现一组三重峰, 为乙酯基中甲基的 吸收峰, 偶合常数为 $7.0 \mathrm{~Hz}$, 在 $\delta 4.24 \sim 4.12$ 处出现多重 峰, 为乙酯基中亚甲基的吸收峰. C(2)不同酯基取代的 杂草 $\mathbf{6 h} \sim \mathbf{6 0}$ 的 ${ }^{1} \mathrm{H}$ NMR 谱中, 分别出现 $\mathrm{CH}_{3}[\delta 3.71(\mathrm{~s})]$, $\mathrm{CH}_{2} \mathrm{CH}_{2} \mathrm{CH}_{3}\left[\delta 4.14 \sim 4.03\left(\mathrm{~m}, \mathrm{OCH}_{2}\right), 1.69 \sim 1.61(\mathrm{~m}\right.$, $\left.\left.\mathrm{CCH}_{2} \mathrm{C}\right), 0.94\left(\mathrm{t}, J=7.5 \mathrm{~Hz}, \mathrm{CCH}_{3}\right)\right], \mathrm{CH}\left(\mathrm{CH}_{3}\right)_{2}[\delta 5.10 \sim$ $5.03\left(\mathrm{~m}, \mathrm{OCH}(\mathrm{C})_{2}\right), 1.26$ (d, $\left.J=6.0 \mathrm{~Hz}, \mathrm{CCH}_{3}\right), 1.20$ (d, $\left.\left.J=6.5, \mathrm{CCH}_{3}\right)\right]$ 和 $\mathrm{C}\left(\mathrm{CH}_{3}\right)_{3}\left[\delta 1.45\left(\mathrm{~s}, \mathrm{OC}\left(\mathrm{CH}_{3}\right)_{3}\right]\right.$ 的吸收 峰, 从而证明上述基团的存在. 在 IR 谱中, 特征 IR 吸收 峰分别是 $1730\left(\mathrm{CO}_{2}\right)$ 和 $1610(\mathrm{~N}=\mathrm{C}) \mathrm{cm}^{-1}$. MS (HRMS) 及元素分析数据也与氧氮杂草 6 的结构相符.

\section{2 副产物的结构及其生成机理}

在室温下、甲醇作溶剂、冰乙酸作催化剂合成目标 化合物 6 时, 经常产生一种副产物(TLC 监测). 在回流 温度下, 该副产物的生成速度加快, 并且成为主产物 (TLC 监测). 我们用柱层析法将其分离, 并通过 IR, ${ }^{1} \mathrm{H}$ NMR 和 HRMS 以及单晶 $X$ 射线衍射法确定了副产物

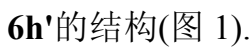<smiles>O=C(CC1Oc2ccccc2NC1=O)c1ccccc1</smiles>

图 1 化合物 $6 h^{\prime}$ 的分子结构

Figure 1 Molecular structure of compound 6h'

\subsection{1 副产物 $6 \mathbf{h}^{\prime}$ '波谱性质及晶体结构}

副产物 $6 \mathbf{h}^{\prime}$ 的核磁共振氢谱中在化学位移 $(\delta)$ 为 10.76 处有一单峰, 属于内酰胺官能团中 $(\mathrm{CO}-\mathrm{NH}-\mathrm{C})$ 氢的化学位移, 与通常内酰胺氢 $(\mathrm{CONH})$ 的化学位移值
相比，该 $\delta$ 值向低场有较大移动; 在 $\delta$ 值为 $8.02 \sim 6.85$ 处有一组 9 个氢的多重峰, 属于苯环上氢的化学位移; 六元环上的一个氢 $(\mathrm{OCHC}=\mathrm{O})$ 和侧链上亚甲基的两个 氢 $\left(\mathrm{CCH}_{2} \mathrm{C}=\mathrm{O}\right)$ 构成 $\mathrm{ABX}$ 系统, 为 12 重峰, 其 $\delta$ 值分别 是 $5.10,3.72,3.67 .6 \mathbf{h}^{\prime}$ 的红外光谱中有三个特征峰 $\left(3194.45,1692.60,1664.55 \mathrm{~cm}^{-1}\right)$, 分别为 $\mathrm{N}-\mathrm{H}$ 键和酰 胺基以及酮羰基的吸收峰. 高分辨质谱显示该化合物的 分子量为 268.09737 ，与其理论值吻合.

晶体结构分析表明，化合物 $\mathbf{6 h}$ '属于单斜晶系, $C 2 / c$ 空间群, 分子式为 $\mathrm{C}_{16} \mathrm{H}_{13} \mathrm{NO}_{3}$, 分子量 $M_{\mathrm{r}} 267.27$, 晶胞 参数: $a=1.2650(2) \mathrm{nm}, b=0.85292(16) \mathrm{nm}, c=1.2685(2)$ $\mathrm{nm}, \alpha=90^{\circ}, \beta=106.429(2)^{\circ}, \gamma=90^{\circ}, V=1.3127(4) \mathrm{nm}^{3}$, $D_{\mathrm{c}}=1.352 \mathrm{Mg} / \mathrm{m}^{3}, Z=4, \mu=0.094 \mathrm{~mm}^{-1}, F(000)=560$, 最后一致性因子 $[I>2 \sigma(I)], R_{1}=0.051, w R_{2}=0.1182$.

由晶体结构图(图 2)以及晶体数据可知, 该副产物 由一个氧氮六元杂环和一个苯环稠合而成, 并且存在分 子内氢键 $[\mathrm{N}(1)-\mathrm{H}=0.89 \mathrm{~nm}, \mathrm{H} \cdots \mathrm{O}(2)=2.01 \mathrm{~nm}, \mathrm{~N} \cdots$ $\left.\mathrm{O}(2)=2.88(16) \mathrm{nm}, \mathrm{N}(1)-\mathrm{H} \cdot \cdots \mathrm{O}(2)=167.3^{\circ}\right]$. 化合物的 单晶 $\mathrm{X}$ 射线分析结果和元素分析、光谱分析 $\left(\mathrm{IR},{ }^{1} \mathrm{H}\right.$ NMR 和 HRMS 谱)结果一致.

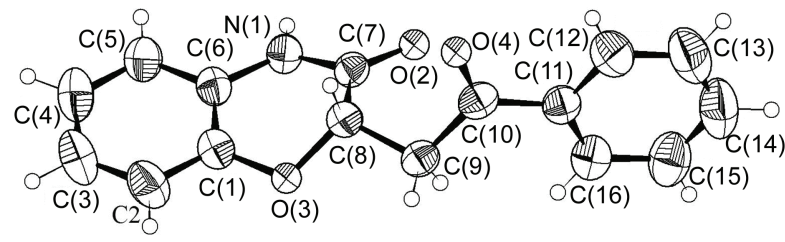

图 2 化合物 $\mathbf{6 h} \mathbf{h}^{\prime}$ 的晶体结构

Figure 2 Crystal structure of compound $\mathbf{6} \mathbf{h}^{\prime}$

\subsection{2目标化合物及副产物生成机理}

目标化合物及副产物的生成机理如 Scheme 2 所示.<smiles>CC(=O)C=CC(=O)c1ccccc1</smiles><smiles>Nc1ccccc1O</smiles>

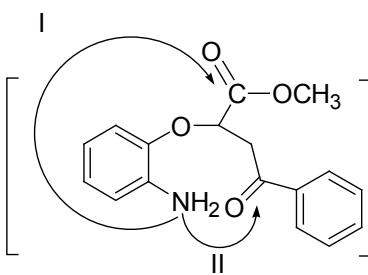

5

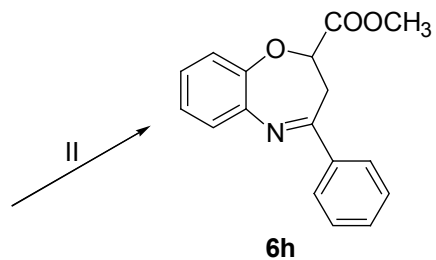

$6 h$

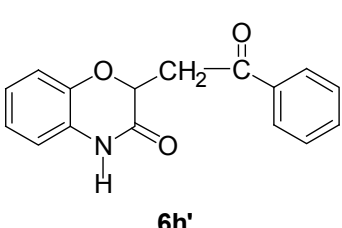

Scheme 2 
首先, 邻氨基苯酚与 $\alpha, \beta$-不饱和化合物 4 发生迈克 尔加成反应, 生成中间体 4-氧代-4-芳基-2-(2-氨基苯氧 基)丁酸酯和 4-氧代-4-芳基-2-(2-氨基-4-氯苯氧基)丁酸 酯(5), 该化合物 5 中氨基与分子内的酯基反应生成酰 胺, 即副产物 $6 h^{\prime}$, 氨基与分子中的酮羰基反应, 生成目 标化合物 $\mathbf{6 h}$, 即氧氮杂草.

\subsection{3 副产物生成条件}

生成 $6 \mathbf{h}$ 和 $6 \mathbf{h}^{\prime}$ 的反应为一对竞争反应. 研究表明, 副产物 $6 \mathbf{h}^{\prime}$ 的产生主要受溶剂的影响. 以甲醇为溶剂、 室温下反应时, 副产物 $\mathbf{6 h}$ '成为主要产物, 但在 DMF 中 几乎没有 $\mathbf{6} \mathbf{h}^{\prime}$ 生成. 在二氯甲烷、苯、乙腈中反应时没 有氧氮杂草 $6 a \sim 60$ 产生, 只有少量的 $6 h^{\prime}$ '生成, 反应进 行的程度也不及在甲醇中完全. 研究结果表明, 在甲 醇、二氯甲烷、苯、乙腈 4 种溶剂中 $\alpha, \beta$-不饱和化合物 4 与氨基苯酚可以发生迈克尔加成, 但当反应进行到一 定程度后, 生成副产物 $\mathbf{6 h}^{\prime}$ '的反应占据了主导地位, 而 在 DMF 中则相反. 另一个影响因素是反应温度, 加热 既有利于氧氮杂草的生成, 也提高了生成副产物的反应 速度.

\section{2 结论}

本文从取代苯、丁烯二酸䣶、醇出发, 合成中间体 $\alpha, \beta$-不饱和化合物 4 , 后者与邻氨基/氯代邻氨基苯酚反 应合成了目标化合物- 1,5 -苯并氧氮杂草 6 . 研究表 明, 生成氧氮杂草 $\mathbf{6}$ 及副产物 $6 h^{\prime}$ 的反应是一对受溶剂 和催化剂影响的竞争反应, 以对甲基苯磺酸为催化剂、 DMF 作溶剂有利于氧氮杂草 $\mathbf{6}$ 的生成, 而以冰醋酸为催 化剂、甲醇作溶剂则有利于副产物 $\mathbf{6 h}$ '的生成.

\section{3 实验部分}

\section{1 仪器与试剂}

X-4 型数字显示显微熔点测定仪(温度计未校正); Vertex 70 FT-IR 型红外分光光度计, 采用 $\mathrm{KBr}$ 压片测得 红外光谱; AVIII500Q Bruker 超导核磁共振仪, $\mathrm{CDCl}_{3}$, DMSO- $d_{6}$ 为溶剂; MT-3 型元素分析仪; xevo 型质谱仪; Waters 2998 高分辨质谱仪; 德国 Bruk-APEX-IIX 射线 单晶衍射仪; varioELIII 型元素分析仪.

所用试剂均为分析纯, 大部分试剂用常规方法干 燥.

\section{2 -位酯基取代-1,5-苯并氧氮杂草 6 的合成}

\subsubsection{4-芳基-4-氧代-2-丁烯酸(3)的合成通法}

向 $250 \mathrm{~mL}$ 四颈瓶中加入 $50 \mathrm{~mL} \mathrm{CH}_{2} \mathrm{Cl}_{2}$, 降温至 0 $-5{ }^{\circ} \mathrm{C}$, 加入 $32.0 \mathrm{~g}(242.6 \mathrm{mmol})$ 无水 $\mathrm{AlCl}_{3}, 8.0 \mathrm{~g}(81.6$ $\mathrm{mmol}$ )的顺丁烯二酸酐, 搅拌下滴加 $81.6 \mathrm{mmol}$ 芳烃 $\mathbf{1}$,
滴加完毕后, 在 $0 \sim-5{ }^{\circ} \mathrm{C}$ 下充分摚拌 $5 \mathrm{~h}$, 停止反应, 缓慢滴加 $100 \mathrm{~mL} 3.5 \mathrm{~mol} / \mathrm{L} \mathrm{HCl}$, 搅拌 $1 \mathrm{~h}$, 将反应液倒 入冰水中, 静置、过滤上述液体得到 $\mathbf{3}$ 的粗品, 向 $\mathbf{3}$ 中 滴加适量饱和 $\mathrm{Na}_{2} \mathrm{CO}_{3}$ 溶液, 煮沸, $\mathrm{pH}$ 值为 $7 \sim 8$. 热过 滤除去杂质，再向滤液中滴加浓盐酸调节 $\mathrm{pH}$ 值至 1 , 过 滤得化合物 3.

\subsubsection{4 芳基-4-氧代-2-丁烯酸酯(4)的合成通法}

在装有分水器的 $100 \mathrm{~mL}$ 圆底烧瓶中加入 28.6 mmol 3、40 mL 苯、 $15 \mathrm{~mL}$ 醇, 滴加 3 滴浓硫酸作催化 剂. 加热回流 $5 \mathrm{~h}$, 停止反应. 待反应液冷却后, 滴加饱 和 $\mathrm{NaHCO}_{3}$ 调节溶液的 $\mathrm{pH}$ 值为 $7 \sim 8$, 静置分液, 用无 水乙醚 $(30 \mathrm{~mL} \times 2)$ 萃取饱和 $\mathrm{NaHCO}_{3}$ 层, 依次用饱和食 盐水和蒸馏水洗有机相, 加无水 $\mathrm{MgSO}_{4}$ 干燥, 过滤, 浓 缩得油状物 4.

4-芳基-4-氧代-2-丁烯酸叔丁酯的合成方法: 在 250 $\mathrm{mL}$ 三颈瓶中加入 $45 \mathrm{mmol}$ 化合物 3, $80 \mathrm{~mL}$ 甲苯, 加入 适量无水 $\mathrm{MgSO}_{4}$ 作为吸水剂, 加入 $15 \mathrm{~mL}$ 叔丁醇, 摚拌 条件下滴加 5 滴浓硫酸, 室温搅拌 $24 \mathrm{~h}$, 反应结束后抽 滤, 再加饱和 $\mathrm{NaHCO}_{3}$ 调溶液 $\mathrm{pH}$ 为 $7 \sim 8$. 静置分液, 用 无水乙醚 $(40 \mathrm{~mL} \times 2)$ 萃取饱和 $\mathrm{NaHCO}_{3}$ 层, 依次用饱和 食盐水和蒸馏水洗有机相, 加无水 $\mathrm{MgSO}_{4}$ 干燥, 过滤, 浓缩, 得到黄色油状物 4-芳基-4-氧代-2-丁烯酸叔丁酯.

\section{2 .3 化合物 6 的合成方法 1(以 $6 \mathrm{~b}$ 为例)}

在 $100 \mathrm{~mL}$ 圆底烧瓶中加入 $7.35 \mathrm{mmol} 4$, 加 $40 \mathrm{~mL}$ 新蒸甲醇将其溶解, 再加入 $0.80 \mathrm{~g}(7.35 \mathrm{mmol})$ 邻氨基苯 酚, 滴加 $0.18 \mathrm{~g}(2.94 \mathrm{mmol})$ 冰乙酸, 加热回流, TLC 监 测反应进程, 约 $30 \mathrm{~h}$ 后停止反应, 柱层析分离得到化合 物 6b, 其结构经 IR, ${ }^{1} \mathrm{H}$ NMR, MS 和元素分析测试确证.

\section{2 .4 化合物 6 的合成方法 2 (以 $6 \mathrm{~b}$ 为例)}

在 $100 \mathrm{~mL}$ 圆底烧瓶中加入 $7.35 \mathrm{mmol}$, 加 $40 \mathrm{~mL}$ 新蒸 DMF 将其溶解, 再加入 $0.80 \mathrm{~g}(7.35 \mathrm{mmol})$ 邻氨基 苯酚, 加 $0.32 \mathrm{~g}(1.84 \mathrm{mmol})$ 对甲苯磺酸, 加热回流, TLC 监测反应进程, 约 $8 \mathrm{~h}$ 后停止反应, 加入 $40 \mathrm{~mL}$ 水, 并用 乙梄多次萃取水层, 至 TLC 监测乙醚层中所含化合物 6 的量很少为止, 加无水 $\mathrm{MgSO}_{4}$ 干燥, 过滤, 浓缩得到黄 色油状物, 经柱层析分离得到化合物 $\mathbf{6 b}$, 其结构经 IR, ${ }^{1} \mathrm{H}$ NMR, MS 和元素分析测试确证.

合成化合物 $6 \mathrm{a} \sim 6 \mathrm{k}$ 时中间体 4 与对甲苯磺酸的物 质的量之比为 $4: 1$, 合成 $\mathbf{6 1} \sim \mathbf{6 0}$ 时二者的物质的量之 比为 $2: 1$.

2,3-二氢-4-(4-甲氧基苯基)-2-乙酯基-1,5-苯并氧氮 杂草 $(6 a)$ : 淡黄色固体, 产率 21.3\%. m.p. 89 91 ${ }^{\circ} \mathrm{C} ;{ }^{1} \mathrm{H}$ NMR $\left(\mathrm{CDCl}_{3}, 500 \mathrm{MHz}\right) \delta: 7.99 \sim 6.91(\mathrm{~m}, 8 \mathrm{H}, \mathrm{PhH})$, $5.93(\mathrm{dd}, J=3.0,10.0 \mathrm{~Hz}, 1 \mathrm{H}, \mathrm{OCH}), 4.24 \sim 4.12(\mathrm{~m}, 2 \mathrm{H}$, $\left.\mathrm{OCH}_{2}\right), 3.88\left(\mathrm{~s}, 3 \mathrm{H}, \mathrm{OCH}_{3}\right), 2.84(\mathrm{dd}, J=10.0,15.5 \mathrm{~Hz}$, 
1H, CH), 2.49 (dd, $J=3.0,15.5 \mathrm{~Hz}, 1 \mathrm{H}, \mathrm{CH}), 1.26(\mathrm{t}, J=$ 7.0, 3H, $\left.\mathrm{CCH}_{3}\right)$; IR (KBr) v: 1723.44, $1606.01 \mathrm{~cm}^{-1}$; MS (EI, $70 \mathrm{eV}) \mathrm{m} / z(\%): 325.95\left([\mathrm{M}+\mathrm{H}]^{+}, 100.0\right)$. Anal. calcd for $\mathrm{C}_{19} \mathrm{H}_{19} \mathrm{O}_{4} \mathrm{~N}$ : C 70.15, H 5.87, N 4.30; found $\mathrm{C} 70.14, \mathrm{H}$ $5.89, \mathrm{~N} 4.31$.

2,3-二氢-4-苯基-2-乙酯基-1,5-苯并氧氮杂草 $(6 \mathbf{b})$ : 淡黄色固体，产率 32.6\%. m.p. $72 \sim 74{ }^{\circ} \mathrm{C} ;{ }^{1} \mathrm{H} \mathrm{NMR}$ $\left(\mathrm{CDCl}_{3}, 500 \mathrm{MHz}\right) \delta: 8.03 \sim 6.93(\mathrm{~m}, 9 \mathrm{H}, \mathrm{PhH}), 5.97$ (dd, $J=2.5,10.0 \mathrm{~Hz}, 1 \mathrm{H}, \mathrm{OCH}), 4.24 \sim 4.12\left(\mathrm{~m}, 2 \mathrm{H}, \mathrm{OCH}_{2}\right)$, $2.86(\mathrm{dd}, J=10.5,15.5 \mathrm{~Hz}, 1 \mathrm{H}, \mathrm{CH}), 2.48(\mathrm{dd}, J=2.0$, $16.0 \mathrm{~Hz}, 1 \mathrm{H}, \mathrm{CH}), 1.26$ (t, $\left.J=7.0 \mathrm{~Hz}, 3 \mathrm{H}, \mathrm{CCH}_{3}\right)$; IR (KBr) v: 1727.57, $1615.27 \mathrm{~cm}^{-1}$; MS (EI, $\left.70 \mathrm{eV}\right) \mathrm{m} / z(\%)$ : $296.20\left([\mathrm{M}+\mathrm{H}]^{+}, 100.0\right)$. Anal. calcd for $\mathrm{C}_{18} \mathrm{H}_{17} \mathrm{O}_{3} \mathrm{~N}$ : C 73.18, H 5.77, N 4.75; found C 73.20, H 5.80, N 4.74.

2,3-二氢-4-(4-甲基苯基)-2-乙酯基-1,5-苯并氧氮杂 草(6c): 淡黄色固体, 产率 31.8\%. m.p. 98 $100{ }^{\circ} \mathrm{C} ;{ }^{1} \mathrm{H}$ NMR $\left(\mathrm{CDCl}_{3}, 500 \mathrm{MHz}\right) \delta: 7.29 \sim 6.92(\mathrm{~m}, 8 \mathrm{H}, \mathrm{PhH})$, $5.95(\mathrm{dd}, J=3.0,10.5 \mathrm{~Hz}, 1 \mathrm{H}, \mathrm{OCH}), 4.24 \sim 4.12(\mathrm{~m}, 2 \mathrm{H}$, $\left.\mathrm{OCH}_{2}\right), 2.84(\mathrm{dd}, J=10.0,15.5 \mathrm{~Hz}, 1 \mathrm{H}, \mathrm{CH}), 2.45$ (dd, $J=$ 3.0, $15.5 \mathrm{~Hz}, 1 \mathrm{H}, \mathrm{CH}), 2.42\left(\mathrm{~s}, 3 \mathrm{H}, \mathrm{PhCH}_{3}\right), 1.26$ (t, $J=7.0$ $\mathrm{Hz}, 3 \mathrm{H}, \mathrm{CCH}_{3}$ ); IR (KBr) v: $1723.44,1609.78 \mathrm{~cm}^{-1}$; MS (EI, $70 \mathrm{eV}) \mathrm{m} / z(\%): 310.32\left([\mathrm{M}+\mathrm{H}]^{+}, 100.0\right)$. Anal. calcd for $\mathrm{C}_{19} \mathrm{H}_{19} \mathrm{O}_{3} \mathrm{~N}$ : C 73.76, H 6.17, N 4.50; found $\mathrm{C} 73.77, \mathrm{H}$ $6.19, \mathrm{~N} 4.53$.

2,3-二氢-4-(4-乙基苯基)-2-乙酯基-1,5-苯并氧氮杂 草(6d): 淡黄色固体, 产率 30.8\%. m.p. 43 45 ${ }^{\circ} \mathrm{C} ;{ }^{1} \mathrm{H}$ NMR $\left(\mathrm{CDCl}_{3}, 500 \mathrm{MHz}\right) \delta: 7.94 \sim 6.91(\mathrm{~m}, 8 \mathrm{H}, \mathrm{PhH})$, $5.94(\mathrm{dd}, J=3.0,10.5 \mathrm{~Hz}, 1 \mathrm{H}, \mathrm{OCH}), 4.24 \sim 4.12(\mathrm{~m}, 2 \mathrm{H}$, $\left.\mathrm{OCH}_{2}\right), 2.84(\mathrm{dd}, J=10.0,15.5 \mathrm{~Hz}, 1 \mathrm{H}, \mathrm{CH}), 2.71$ (q, $J=$ $\left.7.5 \mathrm{~Hz}, 2 \mathrm{H}, \mathrm{PhCH}_{2}\right), 2.46(\mathrm{dd}, J=3.0,15.5 \mathrm{~Hz}, 1 \mathrm{H}, \mathrm{CH})$, $1.28 \sim 1.24\left(\mathrm{~m}, 6 \mathrm{H}, \mathrm{CH}_{3}\right)$; IR $(\mathrm{KBr}) v: 1731.07,1611.08$ $\mathrm{cm}^{-1}$; MS (EI, $\left.70 \mathrm{eV}\right) \mathrm{m} / z(\%): 324.17\left([\mathrm{M}+\mathrm{H}]^{+}, 100.0\right)$. Anal. calcd for $\mathrm{C}_{20} \mathrm{H}_{21} \mathrm{O}_{3} \mathrm{~N}$ : C 74.26, $\mathrm{H}$ 6.52, N 4.31; found C 74.28, H 6.55, N 4.33.

2,3-二氢-4-(4-氟代苯基)-2-乙酯基-1,5-苯并氧氮杂 草(6e): 白色固体, 产率 32.3\%. m.p. 45 46 ${ }^{\circ} \mathrm{C} ;{ }^{1} \mathrm{H}$ NMR $\left(\mathrm{CDCl}_{3}, 500 \mathrm{MHz}\right) \delta: 8.04 \sim 6.93(\mathrm{~m}, 8 \mathrm{H}, \mathrm{PhH})$, $5.92(\mathrm{dd}, J=3.0,10.0 \mathrm{~Hz}, 1 \mathrm{H}, \mathrm{OCH}), 4.24 \sim 4.12(\mathrm{~m}, 2 \mathrm{H}$, $\left.\mathrm{OCH}_{2}\right), 2.85(\mathrm{dd}, J=10.5,16.0 \mathrm{~Hz}, 1 \mathrm{H}, \mathrm{CH}), 2.44(\mathrm{dd}, J=$ 3.5, $15.5 \mathrm{~Hz}, 1 \mathrm{H}, \mathrm{CH}), 1.26$ (t, $\left.J=7.0 \mathrm{~Hz}, 3 \mathrm{H}, \mathrm{CCH}_{3}\right)$; IR (KBr) $v$ : 1726.04, $1606.01 \mathrm{~cm}^{-1}$; MS (EI, $\left.70 \mathrm{eV}\right) \mathrm{m} / z(\%)$ : $314.28\left([\mathrm{M}+\mathrm{H}]^{+}, 100.0\right)$; HRMS-FAB calcd for $\mathrm{C}_{18} \mathrm{H}_{16^{-}}$ $\mathrm{O}_{3} \mathrm{NF}[\mathrm{M}+\mathrm{H}]^{+}: 314.1188$, found 314.1192 .

2,3-二氢-4-(4-氯代苯基)-2-乙酯基-1,5-苯并氧氮杂 草(6f): 淡黄色固体, 产率 32.8\%. m.p. $68 \sim 70{ }^{\circ} \mathrm{C} ;{ }^{1} \mathrm{H}$
NMR $\left(\mathrm{CDCl}_{3}, 500 \mathrm{MHz}\right) \delta: 7.97 \sim 6.92(\mathrm{~m}, 8 \mathrm{H}, \mathrm{PhH})$, $5.91(\mathrm{dd}, J=3.0,10.0 \mathrm{~Hz}, 1 \mathrm{H}, \mathrm{OCH}), 4.23 \sim 4.13(\mathrm{~m}, 2 \mathrm{H}$, $\left.\mathrm{OCH}_{2}\right), 2.85(\mathrm{dd}, J=10.0,16.0 \mathrm{~Hz}, 1 \mathrm{H}, \mathrm{CH}), 2.43(\mathrm{dd}, J=$ 3.0, $15.5 \mathrm{~Hz}, 1 \mathrm{H}, \mathrm{CH}), 1.26$ (t, $J=7.0 \mathrm{~Hz}, 3 \mathrm{H}, \mathrm{CCH}_{3}$ ); IR (KBr) $v: 1732.55,1613.21 \mathrm{~cm}^{-1}$; MS (EI, $\left.70 \mathrm{eV}\right) \mathrm{m} / z(\%)$ : $330.30\left([\mathrm{M}+\mathrm{H}]^{+}, 100.0\right)$. Anal. calcd for $\mathrm{C}_{18} \mathrm{H}_{16} \mathrm{O}_{3} \mathrm{NCl}$ : $\mathrm{C}$ 65.55, H 4.86, N 4.28; found C 65.56, H 4.89, N4.25.

2,3-二氢-4-(4-溴代苯基)-2-乙酯基-1,5-苯并氧氮杂 草(6g): 黄色固体，产率 34.1\%. m.p. $100 \sim 101{ }^{\circ} \mathrm{C} ;{ }^{1} \mathrm{H}$ NMR $\left(\mathrm{CDCl}_{3}, 500 \mathrm{MHz}\right) \delta: 7.89 \sim 6.92(\mathrm{~m}, 8 \mathrm{H}, \mathrm{PhH})$, $5.90(\mathrm{dd}, J=3.0,10.0 \mathrm{~Hz}, 1 \mathrm{H}, \mathrm{OCH}), 4.23 \sim 4.12(\mathrm{~m}, 2 \mathrm{H}$, $\left.\mathrm{OCH}_{2}\right), 2.84(\mathrm{dd}, J=10.0,15.5 \mathrm{~Hz}, 1 \mathrm{H}, \mathrm{CH}), 2.43$ (dd, $J=$ 3.0, $15.5 \mathrm{~Hz}, 1 \mathrm{H}, \mathrm{CH}), 1.26\left(\mathrm{t}, J=7.0 \mathrm{~Hz}, 3 \mathrm{H}, \mathrm{CCH}_{3}\right)$; IR (KBr) v: 1732.26, $1612.39 \mathrm{~cm}^{-1}$; MS (EI, $\left.70 \mathrm{eV}\right) \mathrm{m} / z(\%)$ : $374.50\left([\mathrm{M}+\mathrm{H}]^{+}, 100.0\right)$. Anal. calcd for $\mathrm{C}_{18} \mathrm{H}_{16} \mathrm{O}_{3} \mathrm{NBr}$ : $\mathrm{C}$ 57.75, H 4.32, N 3.72; found C 57.77, H 4.31, N 3.74.

2,3-二氢-4-苯基-2-甲酯基-1,5-苯并氧氮杂草(6h): 淡黄色固体，产率 $32.6 \%$. m.p. 57 59 ${ }^{\circ} \mathrm{C} ;{ }^{1} \mathrm{H} \mathrm{NMR}$ $\left(\mathrm{CDCl}_{3}, 500 \mathrm{MHz}\right) \delta: 8.02 \sim 6.93(\mathrm{~m}, 9 \mathrm{H}, \mathrm{PhH}), 5.97$ (dd, $J=3.0,10.0 \mathrm{~Hz}, 1 \mathrm{H}, \mathrm{OCH}), 3.71\left(\mathrm{~s}, 3 \mathrm{H}, \mathrm{OCH}_{3}\right), 2.87$ (dd, $J=10.5,15.5 \mathrm{~Hz}, 1 \mathrm{H}, \mathrm{CH}), 2.48$ (dd, $J=3.0,15.5 \mathrm{~Hz}, 1 \mathrm{H}$, $\mathrm{CH})$; IR (KBr) v: 1724.20, $1612.40 \mathrm{~cm}^{-1}$; MS (EI, $\left.70 \mathrm{eV}\right)$ $m / z(\%): 282.11\left([\mathrm{M}+\mathrm{H}]^{+}, 100.0\right)$. Anal. calcd for $\mathrm{C}_{17} \mathrm{H}_{15^{-}}$ $\mathrm{O}_{3} \mathrm{~N}$ : C 72.44, H 5.30, N 4.77; found C 72.58, H 5.37, N 4.98 .

2,3-二氢-4-苯基-2-正丙酯基-1,5-苯并氧氮杂草(6i): 白黄色固体，产率 $30.1 \%$. m.p. 42 43 ${ }^{\circ} \mathrm{C} ;{ }^{1} \mathrm{H} \mathrm{NMR}$ $\left(\mathrm{CDCl}_{3}, 500 \mathrm{MHz}\right) \delta: 8.03 \sim 6.92(\mathrm{~m}, 9 \mathrm{H}, \mathrm{PhH}), 5.97$ (dd, $J=2.5,10.5 \mathrm{~Hz}, 1 \mathrm{H}, \mathrm{OCH}), 4.14 \sim 4.03\left(\mathrm{~m}, 2 \mathrm{H}, \mathrm{OCH}_{2}\right)$, $2.87(\mathrm{dd}, J=10.5,16.0 \mathrm{~Hz}, 1 \mathrm{H}, \mathrm{CH}), 2.48$ (dd, $J=3.0$, $15.5 \mathrm{~Hz}, 1 \mathrm{H}, \mathrm{CH}), 1.69 \sim 1.61\left(\mathrm{~m}, 2 \mathrm{H}, \mathrm{CCH}_{2} \mathrm{C}\right), 0.94(\mathrm{t}$, $J=7.5 \mathrm{~Hz}, 3 \mathrm{H}, \mathrm{CCH}_{3}$ ); IR (KBr) v: 1730.00, 1614.30 $\mathrm{cm}^{-1}$; MS (EI, $\left.70 \mathrm{eV}\right) \mathrm{m} / z(\%): 309.94\left([\mathrm{M}+\mathrm{H}]^{+}, 100.0\right)$. Anal. calcd for $\mathrm{C}_{19} \mathrm{H}_{19} \mathrm{O}_{3} \mathrm{~N}$ : C 73.97, $\mathrm{H}$ 6.43, N 4.55; found C 73.77, H 6.19, N 4.53.

2,3-二氢-4-苯基-2-异丙酯基-1,5-苯并氧氮杂草 $(6 \mathbf{j})$ : 白色固体，产率 24.6\%. m.p. 56 58 ${ }^{\circ} \mathrm{C} ;{ }^{1} \mathrm{H} \mathrm{NMR}$ $\left(\mathrm{CDCl}_{3}, 500 \mathrm{MHz}\right) \delta: 8.02 \sim 6.91(\mathrm{~m}, 9 \mathrm{H}, \mathrm{PhH}), 5.95(\mathrm{dd}$, $J=3.0,10.0 \mathrm{~Hz}, 1 \mathrm{H}, \mathrm{OCH}), 5.10 \sim 5.03(\mathrm{~m}, 1 \mathrm{H}$, $\left.\mathrm{OCH}\left(\mathrm{CH}_{3}\right)_{2}\right), 2.82(\mathrm{dd}, J=10.0,15.5 \mathrm{~Hz}, 1 \mathrm{H}, \mathrm{CH}), 2.45$ (dd, $J=3.0,15.5 \mathrm{~Hz}, 1 \mathrm{H}, \mathrm{CH}), 1.26(\mathrm{~d}, J=6.0 \mathrm{~Hz}, 3 \mathrm{H}$, $\left.\mathrm{CCH}_{3}\right), 1.20\left(\mathrm{~d}, J=6.5 \mathrm{~Hz}, 3 \mathrm{H}, \mathrm{CCH}_{3}\right)$; IR (KBr) $v$ : 1722.30, 1616.20 $\mathrm{cm}^{-1}$; MS (EI, $\left.70 \mathrm{eV}\right) \mathrm{m} / z$ (\%): 310.06 $\left([\mathrm{M}+\mathrm{H}]^{+}, 100.0\right)$. Anal. calcd for $\mathrm{C}_{19} \mathrm{H}_{19} \mathrm{O}_{3} \mathrm{~N}$ : C 73.66, $\mathrm{H}$ 6.13, N 4.39; found C 73.77, H 6.19, N 4.53. 
2,3-二氢-4-苯基-2-叔丁酯基-1,5-苯并氧氮杂 草(6k): 白色固体, 产率 4.5\%. m.p. 58 60 ${ }^{\circ} \mathrm{C} ;{ }^{1} \mathrm{H}$ NMR $\left(\mathrm{CDCl}_{3}, 500 \mathrm{MHz}\right) \delta: 8.02 \sim 6.92(\mathrm{~m}, 9 \mathrm{H}, \mathrm{PhH}), 5.92(\mathrm{dd}$, $J=3.0,10.5 \mathrm{~Hz}, 1 \mathrm{H}, \mathrm{OCH}), 2.76(\mathrm{dd}, J=10.5,16.0 \mathrm{~Hz}$, $1 \mathrm{H}, \mathrm{CH}), 2.40$ (dd, $J=3.5,15.5 \mathrm{~Hz}, 1 \mathrm{H}, \mathrm{CH}), 1.45$ (s, 9H, $\left.\mathrm{C}\left(\mathrm{CH}_{3}\right)_{3}\right)$; IR (KBr) $v: 1716.50,1612.40 \mathrm{~cm}^{-1}$; MS (EI, 70 eV) $m / z(\%): 324.08\left([\mathrm{M}+\mathrm{H}]^{+}, 100.0\right)$. Anal. calcd for $\mathrm{C}_{20} \mathrm{H}_{21} \mathrm{O}_{3} \mathrm{~N}$ : C 74.27, $\mathrm{H}$ 6.70, N 4.36; found $\mathrm{C} 74.28, \mathrm{H}$ $6.55, \mathrm{~N} 4.33$.

2,3-二氢-4-苯基-2-甲酯基-7-氯代-1,5-苯并氧氮杂 草(61): 淡黄色固体, 产率 36.7\%. m.p. 93 95 ${ }^{\circ} \mathrm{C} ;{ }^{1} \mathrm{H}$ NMR $\left(\mathrm{CDCl}_{3}, 500 \mathrm{MHz}\right) \delta: 8.01 \sim 6.87(\mathrm{~m}, 8 \mathrm{H}, \mathrm{PhH})$, 5.97 (dd, $J=3.0,10.0 \mathrm{~Hz}, 1 \mathrm{H}, \mathrm{OCH}), 3.72\left(\mathrm{~s}, 3 \mathrm{H}, \mathrm{OCH}_{3}\right)$, 2.84 (dd, $J=10.5,16.0 \mathrm{~Hz}, 1 \mathrm{H}, \mathrm{CH}), 2.48$ (dd, $J=3.0$, $16.0 \mathrm{~Hz}, 1 \mathrm{H}, \mathrm{CH})$; IR (KBr) $v: 1735.80,1616.20 \mathrm{~cm}^{-1}$; MS (EI, $70 \mathrm{eV}) \mathrm{m} / \mathrm{z}(\%): 315.81\left([\mathrm{M}+\mathrm{H}]^{+}, 100.0\right)$. Anal. calcd for $\mathrm{C}_{17} \mathrm{H}_{14} \mathrm{O}_{3} \mathrm{NCl}$ : C 64.92, $\mathrm{H} 4.66, \mathrm{~N} 4.45$; found $\mathrm{C} 64.67$, H 4.47, N 4.44 .

2,3-二氢-4-苯基-2-乙酯基-7-氯代-1,5-苯并氧氮杂 草(6m): 淡黄色固体, 产率 34.5\%. m.p. $76 \sim 77{ }^{\circ} \mathrm{C} ;{ }^{1} \mathrm{H}$ NMR $\left(\mathrm{CDCl}_{3}, 500 \mathrm{MHz}\right) \delta: 8.01 \sim 6.87(\mathrm{~m}, 8 \mathrm{H}, \mathrm{PhH})$, 5.97 (dd, $J=3.0,10.0 \mathrm{~Hz}, 1 \mathrm{H}, \mathrm{OCH}), 4.24 \sim 4.12(\mathrm{~m}, 2 \mathrm{H}$, $\mathrm{OCH}_{2}$ ), 2.82 (dd, $\left.J=10.0,15.5 \mathrm{~Hz}, 1 \mathrm{H}, \mathrm{CH}\right), 2.47$ (dd, $J=$ 3.0, $16.0 \mathrm{~Hz}, 1 \mathrm{H}, \mathrm{CH}), 1.26$ (t, J=7.0 Hz, 3H, $\mathrm{CCH}_{3}$ ); IR (KBr) $v$ : 1739.70, $1612.40 \mathrm{~cm}^{-1}$; HRMS-FAB calcd for $\mathrm{C}_{18} \mathrm{H}_{16} \mathrm{O}_{3} \mathrm{NCl}[\mathrm{M}+\mathrm{H}]^{+}: 330.08941$, found 330.08970 .

2,3-二氢-4-苯基-2-正丙酯基-7-氯代-1,5-苯并氧氮 杂草(6n): 淡黄色固体, 产率 30.5\%. m.p. $48 \sim 49{ }^{\circ} \mathrm{C} ;{ }^{1} \mathrm{H}$ NMR $\left(\mathrm{CDCl}_{3}, 500 \mathrm{MHz}\right) \delta: 8.03 \sim 6.86(\mathrm{~m}, 8 \mathrm{H}, \mathrm{PhH})$, $5.98(\mathrm{dd}, J=3.0,10.5 \mathrm{~Hz}, 1 \mathrm{H}, \mathrm{OCH}), 4.14 \sim 4.03(\mathrm{~m}, 2 \mathrm{H}$, $\mathrm{OCH}_{2}$ ), $2.84(\mathrm{dd}, J=10.0,15.5 \mathrm{~Hz}, 1 \mathrm{H}, \mathrm{CH}), 2.48$ (dd, $J=$ 3.0, $15.5 \mathrm{~Hz}, 1 \mathrm{H}, \mathrm{CH}), 1.69 \sim 1.61\left(\mathrm{~m}, 2 \mathrm{H}, \mathrm{CCH}_{2} \mathrm{C}\right), 0.94$ (t, $J=7.5 \mathrm{~Hz}, 3 \mathrm{H}, \mathrm{CCH}_{3}$ ); IR (KBr) $v: 1728.10,1616.20$ $\mathrm{cm}^{-1}$; MS (EI, $\left.70 \mathrm{eV}\right) \mathrm{m} / \mathrm{z}(\%): 343.83\left([\mathrm{M}+\mathrm{H}]^{+}, 100.0\right)$; $\mathrm{C}_{19} \mathrm{H}_{18} \mathrm{O}_{3} \mathrm{NCl}$ : C 66.63, H 5.47, N 4.08; found C 66.38, H 5.28, N 4.07 .

2,3-二氢-4-苯基-2-异丙酯基-7-氯代-1,5-苯并氧氮 杂草 $(60)$ : 淡黄色固体, 产率 25.3\%. m.p. $51 \sim 52{ }^{\circ} \mathrm{C} ;{ }^{1} \mathrm{H}$ NMR $\left(\mathrm{CDCl}_{3}, 500 \mathrm{MHz}\right) \delta: 8.02 \sim 6.85(\mathrm{~m}, 8 \mathrm{H}, \mathrm{PhH})$, 5.96 (dd, $J=2.5,10.5 \mathrm{~Hz}, 1 \mathrm{H}, \mathrm{OCH}), 5.09 \sim 5.04(\mathrm{~m}, 1 \mathrm{H}$, $\left.\mathrm{CH}\left(\mathrm{CH}_{3}\right)_{2}\right), 2.79(\mathrm{dd}, J=10.5,15.5 \mathrm{~Hz}, 1 \mathrm{H}, \mathrm{CH}), 2.45$ (dd, $J=3.0,15.5 \mathrm{~Hz}, 1 \mathrm{H}, \mathrm{CH}), 1.26\left(\mathrm{~d}, J=6.5 \mathrm{~Hz}, 3 \mathrm{H}, \mathrm{CCH}_{3}\right)$, $1.21\left(\mathrm{~d}, J=6.5 \mathrm{~Hz}, 3 \mathrm{H}, \mathrm{CCH}_{3}\right)$; IR $(\mathrm{KBr}) v: 1724.20$, $1612.40 \mathrm{~cm}^{-1}$; HRMS calcd for $\mathrm{C}_{19} \mathrm{H}_{18} \mathrm{O}_{3} \mathrm{NCl}$ : 344.10524 , found 344.10535 .
2-苯甲酰甲基- $2 H$-1,4-苯并噁溙-3(4H)-酮(6h'): 白 色固体，产率 $63.3 \%$. m.p. $123 \sim 125{ }^{\circ} \mathrm{C} ;{ }^{1} \mathrm{H}$ NMR (DMSO- $\left.d_{6}, 500 \mathrm{MHz}\right) \delta: 10.76(\mathrm{~s}, 1 \mathrm{H}, \mathrm{NH}), 8.02 \sim 6.85$ (m, 9H, PhH), 5.10 (dd, $\left.J=4.5,6.0 \mathrm{~Hz}, \mathrm{OCHCH}_{2}\right), 3.72$ (dd, $J=4.5,18.0 \mathrm{~Hz}, 1 \mathrm{H}, \mathrm{CH}_{2} \mathrm{CO}$ ), 3.67 (dd, $J=6.0,18.0$ $\mathrm{Hz}, 1 \mathrm{H}, \mathrm{CH}_{2} \mathrm{CO}$ ); IR (KBr) v: 3194.45, 1692.60, 1664.55 $\mathrm{cm}^{-1}$; HRMS calcd for $\mathrm{C}_{16} \mathrm{H}_{13} \mathrm{O}_{3} \mathrm{~N}$ : 268.09704, found 268.09737.

\section{3 晶体结构的测定}

选取大小为 $0.48 \mathrm{~mm} \times 0.38 \mathrm{~mm} \times 0.20 \mathrm{~mm}$ 的单晶 6h', 将其置于 rigaku saturn 衍射仪, 经石墨单色器单 色化的 Mo K $\alpha$ 辐射 $(\lambda=0.071073 \mathrm{~nm})$, 采用 $\omega$ 方式扫 描, 在 $298(2) \mathrm{K}$ 温度下, $2.92^{\circ}<\theta<25.50^{\circ}$ 范围内收集数 据, 从衍射区 $-10 \leqslant h \leqslant 15,-9 \leqslant k \leqslant 10,-15 \leqslant l \leqslant 13$ 收集到 6495 个数据, 其中独立衍射点 2442 个 $\left(R_{\mathrm{int}}=\right.$ 0.0299), 单晶结构用 SHELXL 97 结构解析程序直接法 解出, 采用全矩阵最小二乘法进行结构修正, 用直接法 得到全部非氢原子坐标, 所有非氢原子坐标采用各向异 性热参数修正, 所有氢原子均为理论加氢.

\section{References}

[1] Chakravarti, B.; Siddiqui, J. A.; Dwivedi, S. K. D.; Deshpande, S.; Samanta, K.; Bhatta, R. S.; Panda, G.; Prabhaka, Y. S.; Konwar, R.; Sanyal, S.; Chattopadhyay, N. Mol. Cell. Endocrinol. 2011, 338, 68.

[2] Maginn, E. N.; Browne, P. V.; Hayden, P.; Vandenberghe, E.; MacDonagh, B.; Evans, P.; Goodyer, M.; Tewari, P.; Campiani, G.; Butini, S.; Williams, D. C.; Zisterer, D. M.; Lawler, M. P.; McElligott, A. M. Br. J. Cancer 2011, 104, 281.

[3] Zificsak, C. A.; Theroff, J. P.; Aimone, L. D.; Albom, M. S.; Angeles, T. S.; Brown, R. A.; Galinis, D.; Grobelny, J. V.; Herbertz, T.; Husten, J.; Kocsis, L. S.; LoSardo, C.; Miknyoczki, S. J.; Murthy, S.; Rolon-Steele, D.; Underiner, T. L.; Wells-Knecht, K. J.; Worrell, C. S.; Zeigler, K. S.; Dorsey, B. D. Bioorg. Med. Chem. Lett. 2011, 21, 660.

[4] López-Cara, L. C.; Conejo-García, A.; Marchal, J. A.; Macchione, G.; Cruz-López, O.; Boulaiz, H.; García, M. A.; RodríguezSerrano, F.; Ramírez, A.; Cativiela, C.; Jiménez, A. I.; García-Ruiz, J. M.; Choquesillo-Lazarte, D.; Aránega, A.; Campos, J. M. Eur. J. Med. Chem. 2011, 46, 249.

[5] Bajaj, K.; Archana; Kumar, A. Eur. J. Med. Chem. 2004, 39, 369.

[6] Chandrasekhar, S.; Seenaiah, M.; Kumar, A.; Reddy, C. R.; Mamidyala, S. K.; Kumar, C. G.; Balasubramanian, S. Tetrahedron Lett. 2011, 52, 806.

[7] Matsumoto, Y.; Tsuzuki, R.; Matsuhisa, A.; Yoden, T.; Yamagiwa, Y.; Yanagisawa, I.; Shibanuma, T.; Nohira, H. Bioorg. Med. Chem. 2000, 8, 393.

[8] Kamei, K.; Maeda, N.; Nomura, K.; Shibata, M.; Ogino, R. K.; Koyama, M.; Nakajima, M.; Inoue, T.; Ohno, T.; Tatsuoka, T. Bioorg. Med. Chem. 2006, 14, 1978.

[9] Miki, T.; Kori, M.; Fujishima, A.; Mabuchi, H.; Tozawa, R.; Nakamura, M.; Sugiyama, Y.; Yukimasa, H. Bioorg. Med. Chem. 2002, 10, 385.

[10] Miki, T.; Kori, M.; Mabuchi, H.; Banno, H.; Tozawa, R.; Naka- 
mura, M.; Itokawa, S.; Sugiyama, Y.; Yukimasa, H. Bioorg. Med. Chem. 2002, 10, 401.

[11] Miki, T.; Kori, M.; Tozawa, R.; Nakamura, M.; Sugiyama, Y.; Yukimasa, H. Chem. Pharm. Bull. 2002, 50(1), 53.
[12] Ichikawa, M.; Yokomizo, A.; Itoh, M.; Sugita, K.; Usui, H.; Shimizu, H.; Suzuki, M.; Terayama, K.; Kanda, A. Bioorg. Med. Chem. 2011, 19, 1930.

(Zhao, X.) 